\title{
Effects of N-Terminus Substitution on the Structure and Spectroscopy of Gas-Phase Helices
}

\author{
Jaime A. Stearns ${ }^{\S \star}$, Oleg V. Boyarkin, and Thomas R. Rizzo \\ §SCS Mettler-Toledo Award Winner (Oral Presentation)
}

\begin{abstract}
Because of the importance of helices in the secondary structure of proteins, we have undertaken a study of their spectroscopy, fragmentation patterns and conformations in the gas phase. In this work, we describe the effects of substitution at the $\mathrm{N}$-terminus of polyalanine helices capped with a lysine at the C-terminus, namely Ac-Phe(Ala) ${ }_{10}$-Lys- $\mathrm{H}^{+}$, Phe-(Ala) ${ }_{10}-\mathrm{Lys}-\mathrm{H}^{+}$, and $\mathrm{H}^{+}-\mathrm{Phe}-(\mathrm{Ala})_{10}-\mathrm{Lys}^{-\mathrm{H}^{+}}$. Acetylation of the $\mathrm{N}$-terminus has very little effect on the spectroscopy and structure, but protonation of the $\mathrm{N}$-terminus changes the infrared spectrum in such a way that we believe it may also change the structure of the peptide. The ultraviolet spectroscopy and fragmentation, on the other hand, are insensitive to either acetylation or protonation.
\end{abstract}

Keywords: Helices · Gas-phase · Infrared spectroscopy · Photofragmentation · Ultraviolet spectroscopy

\section{Introduction}

Helices are a very common secondary structural element in proteins, and for that reason a significant amount of research is devoted to understanding the intricacies of their folding mechanisms. The gas phase has become an excellent medium for studying the conformational preferences of peptides in recent years as mass spectrometry and laser spectroscopy

${ }^{*}$ Correspondence: Dr. J. A. Stearns

Ecole Polytechnique Fédérale de Lausanne

Laboratoire de Chimie Physique Moléculaire

Station 6

$\mathrm{CH}-1015$ Lausanne

Tel.: +41216933116

Fax: + 41216935170

E-mail: jaime.stearns@epfl.ch combine to provide structural information about increasingly large molecules. Infraredultraviolet (IR-UV) double resonance spectroscopy in a supersonic jet is used by several groups to determine the structural preferences of neutral peptides. ${ }^{[1,2]}$ With the advent of laser desorption, the size of molecules studied using this technique has increased to the point where secondary structure can form. For example, Mons and coworkers have observed 3 -helical structure in two capped tripeptides. ${ }^{[3,4]}$ The study of helices in the gas phase has particularly benefited from the ability of ion mobility spectrometry to distinguish helical from globular structures in larger, charged peptides, which are transferred into the gas phase using laser desorption ionization or electrospray. Using ion mobility, Jarrold and coworkers determined that polyalanines with a lysine at the C-terminus form extremely stable helices in the gas phase. ${ }^{[5,6]}$ The lysine stabilizes the helix by capping the $\mathrm{C}$-terminal carbonyl groups with hydrogen-bonding interactions and by balancing the macrodipole that is formed in the helix by alignment of the amide $\mathrm{NH}$ and $\mathrm{C}=\mathrm{O}$ groups. When the lysine is placed at the N-terminus or is absent, the polyalanine sequence is globular in the gas phase.

We have developed a new technique that combines electrospray ionization with a cold ion trap and IR-UV double resonance spectroscopy to study the structure of gas-phase biomolecules. ${ }^{[7,8]}$ We have recently used this approach to investigate helical peptides of seven and twelve amino acids ${ }^{[9,10]}$ based on the lysine-capped polyalanines used by Jarrold and coworkers. ${ }^{[5,6]}$ The work that we describe here builds on our previous studies with an examination of the effects of the nature of the $\mathrm{N}$-terminus. In most studies of peptides such as those described above, the $\mathrm{N}$-terminus is blocked with an acetyl group to better simulate the intrinsic structure of the sequence as it would be found in the center of a protein. In addition, blocking the $\mathrm{N}$-terminus disfavors protonation at that site because of the lower basicity of the protected amino group relative to the free amine. In gas-phase helices this seems particularly important because of the role of the charged lysine in stabilizing the helix. Here we examine three species of the same amino acid sequence with different $\mathrm{N}$-terminal groups: the protected peptide Ac-Phe-(Ala) 10 -Lys$\mathrm{H}^{+}$, the singly charged unprotected peptide

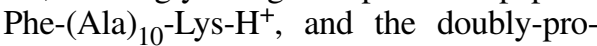
tonated, doubly charged peptide $\mathrm{H}^{+}$-Phe(Ala) ${ }_{10}-\mathrm{Lys}^{-\mathrm{H}^{+}}$. The first of these peptides was included in our initial study ${ }^{[9,10]}$ and is used here as the model of a helical peptide with a protected $\mathrm{N}$-terminus. The other two species are the +1 and +2 charge states of the same peptide with a free $\mathrm{N}$-terminus, allowing us to study the role of a free amino group and a charged ammonium group on the spectroscopy, fragmentation, and conformational preferences of this peptide. 


\section{Experimental Methods}

The apparatus and laser techniques that we employ have been described in previous publications. ${ }^{[7,8]}$ Briefly, we use nanoelectrospray ionization to transfer protonated peptides into the gas phase from a $10^{-4} \mathrm{M}$ solution in methanol, collecting them first in a hexapole ion trap, then passing the resulting ion packet through a quadrupole mass filter to select the peptide of a particular mass-tocharge ratio. A quadrupole deflector turns the ions $90^{\circ}$, where they are decelerated by a series of electrostatic lenses and guided by an rf-only octopole into a cold (6 K), 22-pole ion trap. The ions undergo collisions with cold helium inside the trap, which cools them to $10-15 \mathrm{~K}$. An ultraviolet laser excites the aromatic chromophore of the trapped ions, which causes some fraction of them to dissociate. Lowering the exit electrode of the trap ejects the ions, and a second quadrupole mass filter selects a particular fragment ion for detection. We record UV spectra by monitoring the yield of a particular fragment ion as a function of the UV laser wavenumber, correcting for the total parent ion signal and the laser power. We can also determine the fragmentation pattern due to a particular UV transition by fixing the laser on that transition and scanning the second quadrupole across the desired mass range. Finally, we record IR spectra using a double-resonance depletion method that is conformer-specific. We monitor the constant signal from a particular fragment $\mathrm{m} / \mathrm{z}$ at a particular UV wavelength, but introduce an IR laser approximately 200 ns before the UV. When the IR is resonant with a vibrational transition in the ion, part of the population of that ion is removed to a vibrationally excited state that is usually less efficient at absorbing the UV light, resulting in a depletion in the fragment yield when the IR and UV absorption occur in the same conformer. By monitoring this depletion as a function of IR wavenumber, we obtain a conformation-specific IR spectrum.

The peptides studied here were all synthesized using solid phase Fmoc chemistry on an Applied Biosystems 433 A Synthesizer.

\section{Results and Discussion}

Our approach to studying gas-phase helices requires that we tag the polyalaninelysine peptide with a UV chromophore, which we have chosen to be the natural amino acid phenylalanine. Fig. 1 shows two depictions of Phe-(Ala) ${ }_{10}$-Lys- $^{+}$: a molecular model of it as an $\alpha$-helical peptide, and a schematic drawing of the sequence indicating the important types of hydrogen bonding interactions. The $\mathrm{C}_{10}, \mathrm{C}_{13}$, and $\mathrm{C}_{5}$ arrangements refer to the number of atoms covalently connecting the hydrogen and oxygen of an $\mathrm{N}-\mathrm{H} \cdots \mathrm{O}=\mathrm{C}$ hydrogen bond. Helices incorporate mainly interactions of the type $\mathrm{C}_{10}$ and $\mathrm{C}_{13}$.

As Fig. 2 shows, the UV spectrum of the phenylalanine chromophore is remarkably similar in the three species we investigate here. We have already established that the two largest peaks in Fig. 2a are band origins of two conformations of Ac-Phe-(Ala) ${ }_{10^{-}}$ Lys- $\mathrm{H}^{+}{ }^{[9]}$ Similarly, we now assign the largest peak in Fig. $2 \mathrm{~b}$, at $37512 \mathrm{~cm}^{-1}$, as the band origin of Phe-(Ala) ${ }_{10}-\mathrm{Lys}^{-} \mathrm{H}^{+}$, and the corresponding peak in Fig. $2 \mathrm{c}$ at $37480 \mathrm{~cm}^{-1}$ as the band origin of $\mathrm{H}^{+}-\mathrm{Phe}-(\mathrm{Ala})_{10}-\mathrm{Lys}-\mathrm{H}^{+}$. The band origins of bare, protonated phenylalanine ${ }^{[8]}$ and its neutral counterpart ${ }^{[11]}$ also appear in this region, further demonstrating that the phenylalanine chromophore is relatively insensitive to its environment. The band origin in each spectrum appears as the largest member of a Franck-Condon progression in a low-frequency vibrational mode of $8-10 \mathrm{~cm}^{-1}$. Just to the red of the band origin in each spectrum is a hot band of sizable intensity corresponding to this same low- frequency vibration. Using the intensity of the hot bands relative to the band origins, we estimate a vibrational temperature of 8-15 $\mathrm{K}$. The identity of the low-frequency vibrational mode remains unknown; however, it likely corresponds to a collective motion of the entire peptide. As the only low-frequency vibrational coordinate that changes significantly upon electronic excitation, the motion must be rooted in the relationship between the peptide backbone and the phenyl ring. The second band origin in Ac-Phe-(Ala) $10^{-}$ Lys- $\mathrm{H}^{+}, 40 \mathrm{~cm}^{-1}$ above the first, does not occur in either Phe-(Ala) $10^{-} \mathrm{Lys}^{-\mathrm{H}^{+}}$or $\mathrm{H}^{+}$Phe-(Ala) $10^{- \text {Lys- } \mathrm{H}^{+}}$. Because all the major transitions in Figs. $2 \mathrm{~b}$ and $2 \mathrm{c}$ seem to be part of the vibrational progression, we conclude that both unprotected peptides occur as single conformations.

The similarity of the phenylalanine chromophore in these different local environments is also evidenced by the fragmentation patterns of the three peptides in Fig. 3. Although the intensity patterns differ among the three spectra, the fragmentation channels

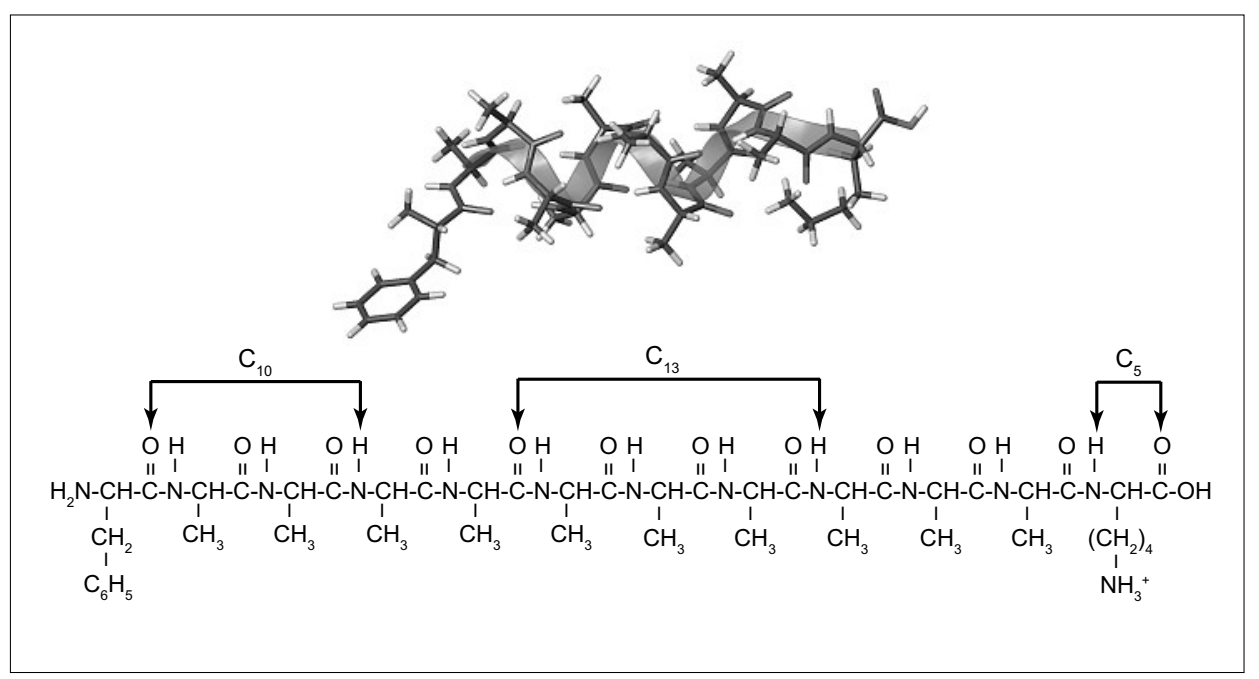

Fig. 1. Presumed helical structure (top) and schematic depiction (bottom) of the singly charged, unprotected peptide Phe-(Ala) ${ }_{10}-\mathrm{Lys}^{-\mathrm{H}^{+}}$. Several important hydrogen-bonding interactions are also shown.

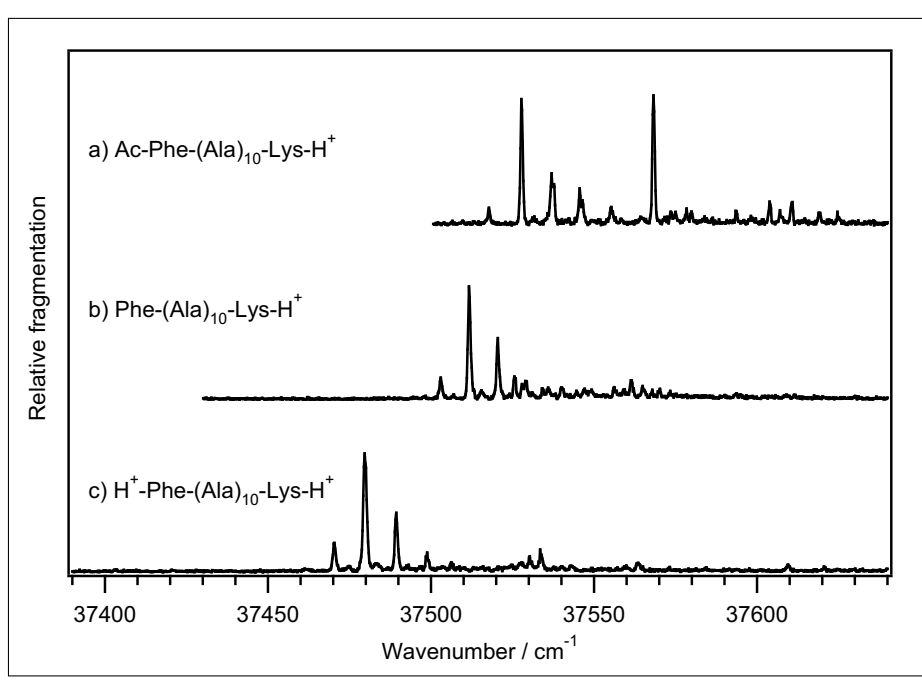

Fig. 2. Ultraviolet photofragmentation spectra in the region of the band origins of a) the $\mathrm{N}$-terminally protected, singly charged Ac-Phe(Ala) ${ }_{10^{-}}$Lys- $\left.\mathrm{H}^{+}, \mathrm{b}\right)$ the singly charged, unprotected Phe(Ala) ${ }_{10}-$ Lys- $^{+}$, and c) the doubly charged, unprotected $\mathrm{H}^{+}$-Phe(Ala) ${ }_{10}{ }^{-L y s}-\mathrm{H}^{+}$. 
are largely the same. The dominant type of fragment involves loss of the neutral phenylalanine side chain radical $\mathrm{C}_{7} \mathrm{H}_{7}$, giving $\mathrm{m} / \mathrm{z}$ 956, 914, and 458 in Figs. 3a-c, respectively. Loss of the acetyl group accompanies this fragmentation in the protected peptide to give $\mathrm{m} / \mathrm{z} 913$ (Fig. 3a). The other fragments that appear in all three peptides are $\mathrm{m} / \mathrm{z} 884$ (442) and $\mathrm{m} / \mathrm{z} 841$ (421) in the singly (doubly) charged species. These fragments arise from cleavage of the peptide backbone at the phenylalanine $\mathrm{C}_{\alpha}-\mathrm{C}_{\text {amide }}$ bond and at the bond between the amide nitrogen and $\mathrm{C}_{\alpha}$ of the first alanine. Two other fragment masses appear in the singly charged peptides, but are less well-defined in $\mathrm{H}^{+}$-Phe-(Ala) ${ }_{10}$-Lys- $\mathrm{H}^{+}$: $\mathrm{m} / \mathrm{z} 813$, from breaking the $\mathrm{C}_{\alpha}-\mathrm{C}_{\text {amide }}$ bond of the first alanine and $\mathrm{m} / \mathrm{z} 858$, from breaking the peptide bond between the phenylalanine and the first alanine. All of the observed fragmentation occurs in the first three residues of the peptides (from the N-terminus) with the charge retained on the larger, $\mathrm{C}$ terminal fragments.

By changing the timing between the UV laser and the release of the ions from the 22-pole trap, we can gauge the time required for the photoexcited molecules to fragment. The flight time of the ions from the 22-pole trap to the detector is on the order of $100 \mu \mathrm{s}$, and represents the lower limit on the fragmentation time we can measure. We observed no change in the fragmentation yield as a function of laser timing, meaning it is faster than $100 \mu \mathrm{s}$, and this suggests that dissociation must occur on an electronically excited state and not via a statistical process in the ground state, which would be significantly slower. ${ }^{[12,13]}$ We also observed excited-state fragmentation in $\mathrm{PheH}^{+}$and the smaller helical peptide Ac-Phe-(Ala) $)_{5}$-Lys- $\mathrm{H}^{+}$. ${ }^{[8,10]}$

In order to establish the three-dimensional structure of these peptides, we use infrared spectroscopy in the $\mathrm{NH}$ and $\mathrm{OH}$ stretch region. Because of their size, we were not able to carry out high-quality geometry and frequency calculations in order to assign the spectra. However, we know from ion mobility studies ${ }^{[5]}$ that these peptides are likely helical, and there are several key features of the infrared spectrum of Ac-Phe-(Ala) ${ }_{10^{-}}$ Lys- $\mathrm{H}^{+}$(Fig. 4a) that we have identified by comparison to Ac-Phe-(Ala) $)_{5}-\mathrm{Lys}_{-} \mathrm{H}^{+}$, for which a detailed analysis was possible using density functional theory calculations. ${ }^{[9,10]}$ The carboxylic acid $\mathrm{OH}$ stretch, which occurs near $3575 \mathrm{~cm}^{-1}$, appears to be free from all hydrogen bonding (see Fig. 1). The amide $\mathrm{NH}$ of the lysine is involved in $\mathrm{a}_{5}$ arrangement, a stabilizing interaction that is not as strong as other hydrogen bonding. The amide $\mathrm{NH}$ groups near the N-terminus of the helix are also not involved in the helical hydrogen bonds, because the first amide $\mathrm{NH}$ that can form at least a $\mathrm{C}_{10}$ ring is the second alanine in an acetylated peptide and the third alanine

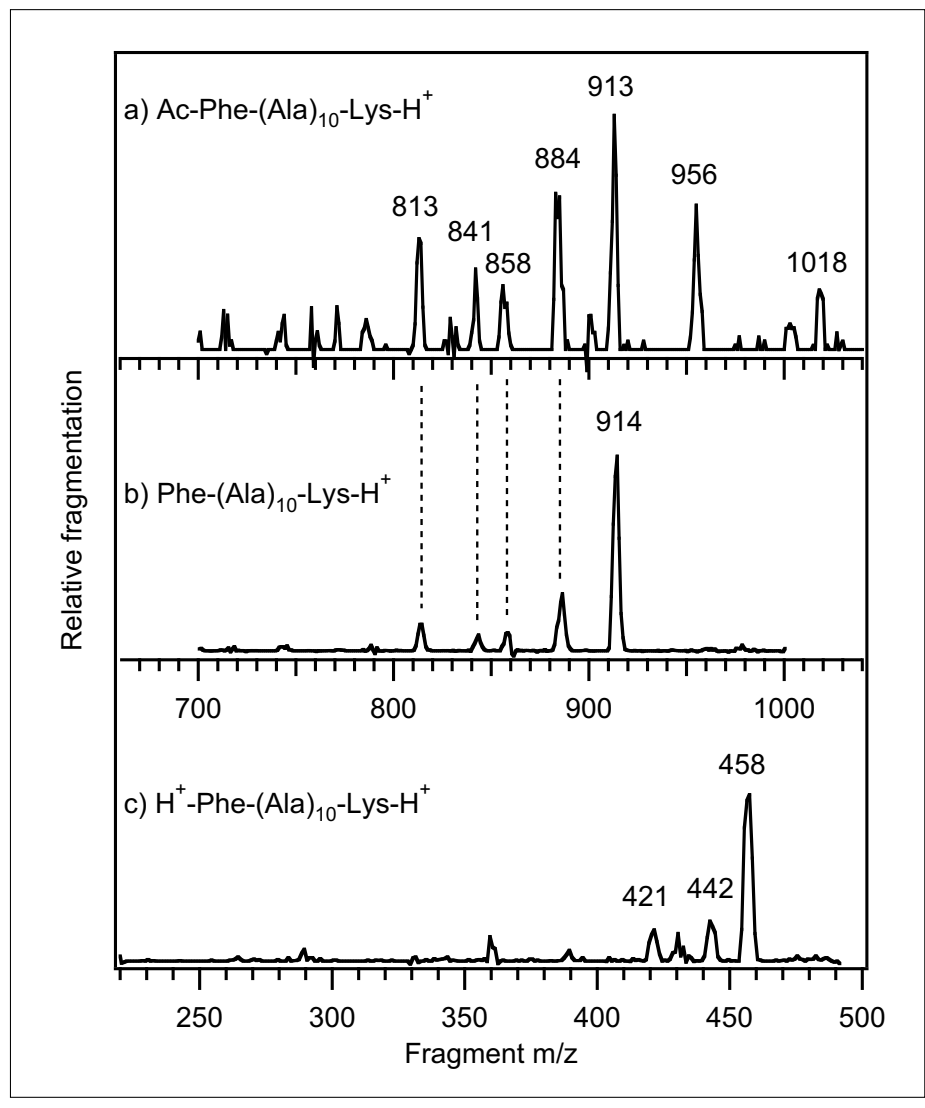

Fig. 3. Fragmentation mass spectra of a) Ac-Phe-(Ala) ${ }_{10}$-Lys- $\mathrm{H}^{+}$, b) Phe-(Ala) ${ }_{10}$-Lys- $\mathrm{H}^{+}$, and c) $\mathrm{H}^{+}$-Phe-(Ala) ${ }_{10^{-}}$ Lys- $\mathrm{H}^{+}$, recorded at the first band origin of each species. The parent mass-to-charge ratios are 1047, 1005, and 503 , respectively. The major fragments in each spectrum are labeled by their massto-charge ratio.

in a peptide with a free N-terminus (Fig. 1). Because they are involved in only weak interactions, the amide NH groups at the ends of the helix have stretching vibrations at frequencies above $\sim 3400 \mathrm{~cm}^{-1}$, although the $\mathrm{NH}$ groups near the phenylalanine may engage in a $\pi$-hydrogen bond with the aromatic ring, which will redshift the frequency slightly. The rest of the amide NH groups are involved in strong hydrogen bonds of either $\mathrm{C}_{10}$ or $\mathrm{C}_{13}$ types. In agreement with calculations on helical glycine-based pentapeptides, ${ }^{[14]}$ we have found that the $\mathrm{NH}$ involved in the $\mathrm{C}_{10}$ ring at the $\mathrm{N}$-terminus of the helix has the strongest redshift and appears near $3300 \mathrm{~cm}^{-1}$. The remaining amide $\mathrm{NH}$ groups appear between $3300 \mathrm{~cm}^{-1}$ and $3400 \mathrm{~cm}^{-1}$, and we have postulated that those falling between 3320 and $3350 \mathrm{~cm}^{-1}$ belong to amide $\mathrm{NH}$ groups in $\alpha$-helical $\mathrm{C}_{13}$ rings.

With this knowledge, we can qualitatively assign the IR spectrum of Phe(Ala) ${ }_{10}-$ Lys- ${ }^{+}$, which is shown in Fig. 4b. The carboxylic acid $\mathrm{OH}$ stretch appears at its free position near $3575 \mathrm{~cm}^{-1}$. The free $\mathrm{NH}$ stretch region has two transitions, which likely correspond to the lysine amide $\mathrm{NH}$, the amide $\mathrm{NH}$ groups from the first two alanines, or the amino group. Those that do not appear in this region are either very weak, or may be shifted further red by a particularly strong $\mathrm{C}_{5}$ or $\pi$-hydrogen bond. The spectra

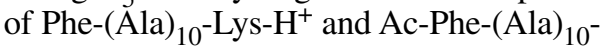
Lys- $\mathrm{H}^{+}$show nearly the same transitions between $3300 \mathrm{~cm}^{-1}$ and $3400 \mathrm{~cm}^{-1}$, which arise from the hydrogen-bonded $\mathrm{NH}$ groups in the center of the helix. The furthest red-shifted transition of Phe-(Ala) ${ }_{10}-\mathrm{Lys}^{-\mathrm{H}^{+}}$likely arises from the $\mathrm{NH}$ group in a $\mathrm{C}_{10}$ arrangement at the N-terminus. The overall similarity between the IR spectra of Ac-Phe-(Ala) ${ }_{10^{-}}$ Lys- $\mathrm{H}^{+}$and Phe-(Ala) ${ }_{10}$-Lys- $\mathrm{H}^{+}$suggests that the unprotected peptide is protonated at the more basic lysine residue rather than the $\mathrm{N}$-terminus, and that these two peptides have very similar helical structures.

The IR spectrum of $\mathrm{H}^{+}$-Phe-(Ala) ${ }_{10}$-Lys$\mathrm{H}^{+}$(Fig. 4c) shares some features with the spectra of the singly charged peptides but is different in some respects. The carboxylic acid $\mathrm{OH}$ stretch appears $\sim 10 \mathrm{~cm}^{-1}$ lower in the doubly charged peptide, but the region around $3450 \mathrm{~cm}^{-1}$ corresponding to weakly interacting amide $\mathrm{NH}$ groups is very similar. The hydrogen-bonded $\mathrm{NH}$ stretch region of the doubly charged peptide in Fig. 4c is substantially different from the other two spectra shown in Fig. 4. Part of this difference comes from the extra ammonium group located on the phenylalanine residue. This part of the peptide now resembles protonated phenylalanine, with the three ammonium hydrogens either free, pointing into the phenyl ring $\pi$ cloud, or in a $\mathrm{C}_{5}$ interaction with the amide carbonyl. ${ }^{[8]}$ In $\mathrm{PheH}^{+}$, the ammonium $\mathrm{NH}$ groups in these types of weaker interactions appeared near $3340 \mathrm{~cm}^{-1},{ }^{[8]}$ so the extra bands below $3300 \mathrm{~cm}^{-1}$ may belong to such groups, redshifted by interaction with the rest of the molecule. The fact that these are broadened substantially, even when compared to the somewhat broad transitions just 


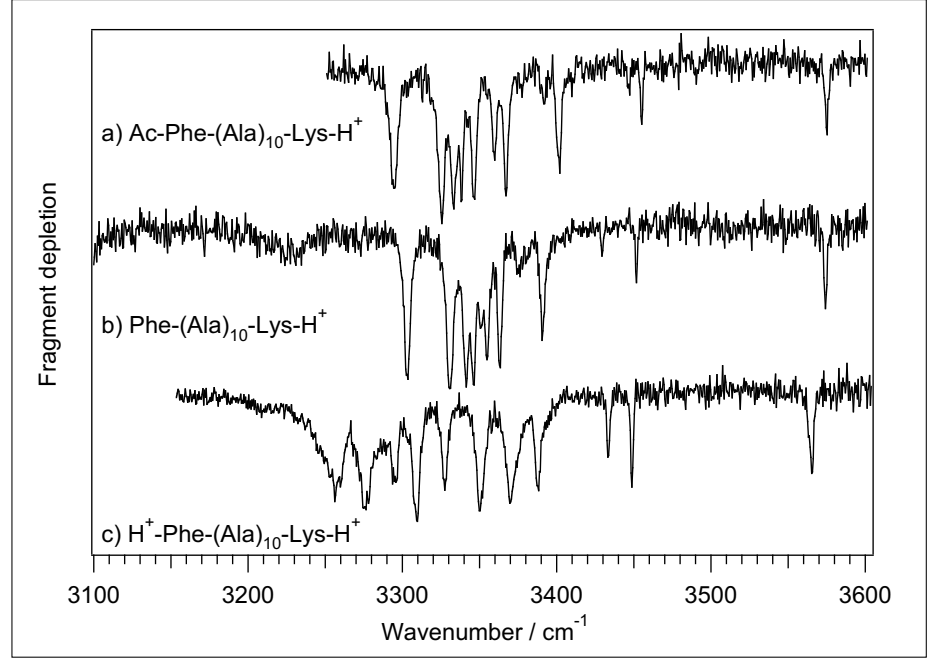

Fig. 4. Conformationspecific infrared spectra of a) Ac-Phe(Ala) ${ }_{10}-$ Lys $^{-\mathrm{H}^{+}}$, b) Phe-(Ala) ${ }_{10}-$ Lys- $\mathrm{H}^{+}$, and c) $\mathrm{H}^{+}-$Phe-(Ala) ${ }_{10^{-}}$ Lys- $\mathrm{H}^{+}$, recorded at the first band origin of each species. above $3300 \mathrm{~cm}^{-1}$, supports our assignment of them as vibrations of ammonium rather than amide NH groups. The pattern between 3300-3400 $\mathrm{cm}^{-1}$ exhibits sparser and broader transitions than in the singly charged peptides shown in Figs. 4a and $4 \mathrm{~b}$. This difference suggests that $\mathrm{H}^{+}$-Phe-(Ala) $)_{10}$-Lys$\mathrm{H}^{+}$may not be completely helical, despite the striking similarity of its UV spectra to those of the helical peptides. This suggestion is supported by the notion that helix formation in the gas phase is driven in part by the balance between the macrodipole of the helix and the positive charge on the lysine. ${ }^{[5]}$ Although that positive charge has not been removed in this case, the additional positive charge at the N-terminus of the peptide counteracts its effect by providing an equal, destabilizing charge. The appearance of the $\mathrm{OH}$ stretch near its normal free position in Fig. $4 \mathrm{c}$ indicates that the $\mathrm{C}$-terminal portion of the peptide may retain a helical structure which prevents the $\mathrm{C}$-terminus from wrapping around to hydrogen-bond to the rest of the peptide. Because of the additional charge at the $\mathrm{N}$-terminus, however, it seems that the helical structure may not persist the entire length of the peptide. It is also possible that the peptide is still helical, but the vibrational frequencies of the hydrogen-bonded amide $\mathrm{N}-\mathrm{H}$ groups are affected by the additional charge such that they fall into a pattern different from those of the singly charged peptides.

We recorded the IR spectrum of each band in the UV spectra of Phe-(Ala) ${ }_{10}$-Lys$\mathrm{H}^{+}$and $\mathrm{H}^{+}$-Phe-(Ala) $10^{-}$-Lys- $\mathrm{H}^{+}$that was sizable enough to accommodate the doubleresonance technique, confirming that every transition belongs to a single conformation for both charge states of the unprotected peptide, even though the protected peptides Ac-Phe-(Ala) $10^{- \text {Lys- }^{+}}$and Ac-Phe-(Ala) $5^{-}$ Lys- $\mathrm{H}^{+}$appear as pairs of conformers differing by the orientation of the phenylalanine ring with respect to the helix axis.[9] We suggest three possible explanations for this.
First, a particular ring orientation might be favored energetically when the acetyl group is removed, such that the other is not populated significantly. However, calculations of the model peptide Phe-(Ala) $)_{5}-\mathrm{Lys}_{-} \mathrm{H}^{+}$indicate that the different ring orientations are only a few $\mathrm{kJ} / \mathrm{mol}$ different in energy, similar to Ac-Phe-(Ala) $)_{5}-\mathrm{Lys}^{-\mathrm{H}^{+}}$. ${ }^{[9]}$ The second possibility is that there is only a small barrier between the two ring orientations in Phe-(Ala) ${ }_{10}-\mathrm{Lys}_{-} \mathrm{H}^{+}$, so that when the ions are cooled in the trap, the population is funneled into the lower-energy conformer. The third possibility is that both conformations are populated at low temperature, but that they have different fragmentation patterns, and only one conformer undergoes fragmentation in an excited electronic state. Because of the size of the peptide, statistical dissociation in the ground state should occur too slowly for us to observe, ${ }^{[12,13]}$ so if a conformation exists that does not undergo excited-state fragmentation, we would be unable to detect it using our UV photofragmentation technique under the conditions we currently employ. Although we observed no conformation-dependent fragmentation in protonated Phe, ${ }^{[8]}$ Ebata and coworkers ${ }^{[15]}$ previously reported conformation-dependent fluorescence lifetimes in neutral Phe. If it is the case that the unprotected $\mathrm{N}$-terminus results in different fragmentation patterns for different conformers, we will be able to observe this difference in a smaller peptide for which we can detect ground state fragmentation, namely Phe-(Ala) $)_{5}$-Lys- $\mathrm{H}^{+}$.

\section{Conclusions}

We have investigated the role of substitution and charge at the N-terminus on the ultraviolet spectroscopy, photofragmentation, and infrared spectroscopy of three

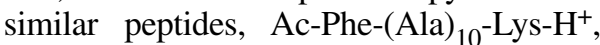

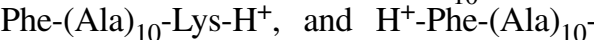
Lys- $\mathrm{H}^{+}$. The UV spectra and fragmentation patterns of each are quite similar, but we observe two stable conformations for the peptide with an acetylated $\mathrm{N}$-terminus while both charge states of the unprotected peptide show a single conformation. We attribute this either to a strong energetic preference for a particular orientation of the phenylalanine ring with respect to the helix axis, or to possible suppression of excited state fragmentation in a particular conformation. The IR spectrum confirms that the unprotected peptide is helical in the +1 charge state, but the +2 charge state may be only partially helical. We conclude that the peptide conformation is influenced little by the presence of the acetyl group but the presence of an extra positive charge may have a greater impact.

\section{Acknowledgement}

We are grateful to Catherine Servis and the University of Lausanne Protein and Peptide Chemistry Facility for synthesis of the peptides. Funding was provided by the École Polytechnique Fédérale de Lausanne and the Fonds National Suisse through grant no. 200020-112071.

Received: February 25, 2008

[1] W. Chin, F. Piuzzi, I. Dimicoli, M. Mons, Phys. Chem. Chem. Phys. 2006, 8, 1033.

[2] M. Gerhards, in 'Principles of Mass Spectrometry Applied to Biomolecules', Eds. J. Laskin, C. Lifshitz, John Wiley \& Sons, Inc, Hoboken, NJ, 2006, p 3.

[3] W. Chin, F. Piuzzi, J. P. Dognon, I. Dimicoli, B. Tardivel, M. Mons, J. Am. Chem. Soc. 2005, 127, 11900 .

[4] V. Brenner, F. Piuzzi, I. Dimicoli, B. Tardivel, M. Mons, J. Phys. Chem. A 2007, 111,7347

[5] R. R. Hudgins, M. F. Jarrold, J. Am. Chem Soc. 1999, 121, 3494.

[6] M. Kohtani, T. C. Jones, J. E. Schneider, M. F. Jarrold, J. Am. Chem. Soc. 2004, 126, 7420.

[7] O. V. Boyarkin, S. R. Mercier, A Kamariotis, T. R. Rizzo, J. Am. Chem. Soc. 2006, 128,2816

[8] J. A. Stearns, S. Mercier, C. Seaiby, M. Guidi, O. V. Boyarkin, T. R. Rizzo, J. Am. Chem. Soc. 2007, 129, 11814

[9] J. A. Stearns, O. V. Boyarkin, T. R. Rizzo, J. Am. Chem. Soc. 2007, 129, 13820.

[10] J. A. Stearns, C. Seaiby, O. V. Boyarkin, T. R. Rizzo, in preparation.

[11] S. J. Martinez III, J. C. Alfano, D. H. Levy, J. Mol. Spectrosc. 1992, 156, 421.

[12] L. L. Griffin, D. J. McAdoo, J. Am. Soc. Mass Spectrom. 1993, 4, 11.

[13] Y. Hu, B. Hadas, M. Davidovitz, B. Balta, C. Lifshitz, J. Phys. Chem. A 2003, 107, 6507.

[14] R. Wieczorek, J. J. Dannenberg, J. Am. Chem. Soc. 2003, 125, 14065.

[15] T. Hashimoto, Y. Takasu, Y. Yamada, T. Ebata, Chem. Phys. Lett. 2006, 421, 227. 